\title{
Hábitos de consumo de embutidos en el cantón de San Carlos y el área metropolitana de Costa Rica
}

\author{
Sausage 's consumer habits in two different \\ geographical areas in Costa Rica
}

Yorleny Araya-Quesada'

Andrea Jiménez-Robles²

Carmen Ivankovich-Guillén ${ }^{3}$

Manuel García-Barquero ${ }^{4}$

Fecha de recepción: 27 de marzo del 2014

Fecha de aprobación: 05 de junio del 2014

Araya-Quesada, Y; Jiménez-Robles, A; Ivankovich-

Guillén, C; García-Barquero, M. Hábitos de

consumo de embutidos en el cantón de San

Carlos y el área metropolitana de Costa Rica.

Tecnología en Marcha. Vol. 27, N 4, Octubre-

Diciembre. Pág | |3-124.

I Escuela de Tecnología de Alimentos, Universidad de Costa Rica. Correo electrónico: yorleny.araya@ucr.ac.cr

2 Maestría Gerencia Agroempresarial, Universidad de Costa Rica. Correo electrónico: andreajrobles@gmail.com

3 Centro Nacional de Ciencia y Tecnología de Alimentos, Universidad de Costa Rica. Correo electrónico: carmen.ivankovich@ucr.ac.cr

4 Escuela de Economía Agrícola y Agronegocios, Universidad de Costa Rica. Correo electrónico: manuel.garcia@ucr.ac.cr 


\section{Palabras clave}

Embutidos; sesiones focales; encuesta; hábitos de consumo; preferencias de consumo.

\section{Resumen}

Se realizó un estudio de mercado en dos áreas geográficas de Costa Rica: San Carlos y la Gran Área Metropolitana, con el fin de comparar los hábitos de consumo de embutidos. En San Carlos se hizo un minigrupo con cinco participantes y un grupo focal con nueve. En el Área Metropolitana se hicieron dos grupos focales con seis personas. Los resultados de las sesiones se analizaron por análisis temático. Se diseñó un cuestionario basado en la investigación cualitativa que se aplicó por teléfono y en el que participaron 193 personas. Los datos se analizaron utilizando estadística descriptiva. Se determinaron las relaciones utilizando una prueba chi-cuadrado $\left(X^{2}\right)$, con significancia del $5 \%$. No se encontraron diferencias significativas en los hábitos entre los consumidores, excepto para el embutido de preferencia, pues en el Área Metropolitana es el jamón, mientras que en San Carlos es el salchichón. Los embutidos se consumen por su buen sabor y por ser convenientes; se suelen acompañar con diversos alimentos.

\section{Key words}

Sausages; focus group; survey; consumer habits; consumer preferences.

\begin{abstract}
Market research was conducted in two different geographical areas in Costa Rica: San Carlos and Metropolitan area in order to compare the consumer preferences about sausages. One mini group session with 5 persons and a group session with 9 persons were conducted in San Carlos. In the Metropolitan area, two mini groups session with 6 persons each also took place. A thematic analysis was applied. A questionnaire was designed using results from qualitative investigation. The survey was by telephone, the sample size was $n=193$. Survey data were analyzed using descriptive statistics and also to relate the results a chi-square ratio test $\left(X^{2}\right)$ was applied (5\% significance). Not significant differences were found between people from the two geographical areas, except sausages preference in the Metropolitan area is ham and in San Carlos is saucisson. Consumers eat sausages because they have good taste and are convenient, are usually accompanied with cereals and vegetables.
\end{abstract}

\section{Introducción}

En Costa Rica, la industria de los embutidos y el procesamiento de carne ha venido evolucionando desde operaciones manuales hasta la situación actual, con el establecimiento de plantas procesadoras con diferentes dimensiones, diversos grados de automatización en sus procesos y condiciones de infraestructura específicas para asegurar productos de calidad e inocuos. En los últimos años, esta preocupación por la calidad de los productos es lo que le ha permitido a las empresas del sector cárnico costarricense experimentar un aumento tanto en el volumen de producción de los alimentos como en la variedad de los embutidos ofrecidos a nivel nacional, entre los que se pueden citar mortadela, salchichón, salchichas, paté, salame, chorizo y jamones (Guillén, 2007). La investigación de mercados consiste en el diseño, la recopilación, el análisis y el reporte de la información y de los datos relevantes del mercado (Kotler y Keller, 2006). Esto ayuda en el proceso de toma de decisiones en mercadeo (Pope, 2002) y posibilita la conexión entre el productor y el consumidor a través de la investigación, ya sea cuantitativa, cualitativa o ambas (Sutherland, 199|). Este tipo de estudio resulta fundamental para conocer las expectativas de los consumidores, de modo que las estrategias de comercialización permitan desarrollar una ventaja competitiva (Benassini, 200 I; Hill y Jones, 2005). Con el objetivo de conocer los hábitos de los consumidores de embutidos de dos áreas geográficas distintas de Costa Rica, se llevó a cabo una investigación de mercado utilizando herramientas cualitativas y cuantitativas. 


\section{Materiales y métodos}

Con el propósito de identificar las diferencias en los hábitos del consumidor con respecto a los embutidos, se compararon dos zonas geográficas de Costa Rica: la Gran Área Metropolitana (GAM), que tiene el 22\% de su población en zona rural; y el cantón de San Carlos, con alrededor del 75\% de su población en zona rural. El estudio se realizó en el periodo de septiembre a noviembre de 20 I I. Se utilizaron herramientas de investigación cualitativas y cuantitativas, modalidad muy aplicada en mercadeo (Seymour, 1988). Es común realizar entrevistas previamente al desarrollo de los cuestionarios o encuestas, para facilitar la comunicación con el potencial consumidor (Bellenger, Bernhardt y Goldstucker, 1976).

\section{Investigación cualitativa}

En mercadeo, la investigación cualitativa se utiliza para conocer las percepciones de los consumidores y lograr un entendimiento real, lo que permite orientar las estrategias y generar satisfacción en los compradores (Gordon y Goldsmith, 1994). El comportamiento del consumidor implica un proceso dinámico que involucra muchas facetas, entre las que se encuentra la motivación para adquirir un producto o servicio (Hoyer y Maclnnis, 20 I0).

Para identificar los temas de mayor interés por parte de los consumidores y así definir los tópicos por tratar en las sesiones grupales, se realizó una entrevista en profundidad semiestructurada, para la cual se diseñó una guía que incluyó diversos aspectos relacionados con el consumo de embutidos. Esta herramienta se aplicó a 12 personas con edades entre 22 y 55 años que residían en la GAM.

Se realizaron dos sesiones grupales, cada una con seis consumidores que habitan en la GAM. En San Carlos se efectuó una minisesión con cinco participantes y una sesión con nueve participantes. En la literatura sobre grupos focales se reportan entre seis y diez participantes (Freitas, Oliveira, Jenkins y Popjoy, 1998), o entre ocho y doce (Fern, 1982); y para el caso de minigrupos, entre tres y cinco participantes.

En su mayoría participaron mujeres laboralmente activas y con edades comprendidas entre 23 y 56 años. En todas las sesiones de grupo se utilizó una guía de sesión en la cual se estableció la agenda y los temas a tratar según los resultados obtenidos en las entrevistas en profundidad. En la guía se incluyeron temas como: tipo de embutidos que se consumen, frecuencia de consumo, formas de preparación y consideraciones al efectuar la compra. En el campo de los estudios en alimentos, las entrevistas en profundidad y las sesiones grupales se utilizan ampliamente como herramientas de investigación (Barrios \& Costell, 2004). El uso de la metodología cualitativa permitió hacer preguntas directamente al segmento meta y solicitar información en detalle sobre el consumo de embutidos. La sesión fue conducida por una moderadora y se contó con el apoyo de un observador, que tomó nota y atendió los detalles logísticos durante la ejecución de la actividad. Al inicio de la sesión, la moderadora indicó los objetivos del estudio y las reglas (Meilgaard, Vance y Carr, 2007). Se grabó el audio de las reuniones, que tuvieron una duración de 70 minutos.

Para el análisis de los resultados se transcribieron las sesiones grabadas. Se hizo un análisis temático en el cual se identificaron los conceptos que los participantes repitieron más veces (Ivankovich-Guillén y Araya-Quesada, 20 I I).

\section{Investigación cuantitativa}

Se realizó una encuesta, para la cual se diseñó una herramienta con preguntas cerradas, principalmente, basada en los resultados de las entrevistas en profundidad y los grupos focales. El tamaño de muestra se calculó considerando un 95\% de confianza, asumiendo un valor de $p$ de 0,5 y de q de 0,5 y un error del 7\%, y correspondió a 193 personas; 97 residentes de la GAM y 96 de San Carlos. El método de contacto fue la vía telefónica. Se utilizó muestreo simple al azar. Los datos se analizaron mediante estadística descriptiva y además, para relacionar las características socio-demográficas, se aplicó la prueba de relación chi-cuadrado $\left(X^{2}\right)$ (5\% de significancia). Para el análisis de los datos se utilizó el programa estadístico JMP, versión 8.0.

\section{Resultados y discusión}

Para la encuesta, la distribución de los participantes por género fue muy similar, ya que el $80 \%$ y el $81 \%$ de la GAM y de San Carlos respectivamente fueron mujeres (cuadro I).

En relación con la edad (cuadro 2), las personas encuestadas fueron distribuidas en cinco grupos, 
donde alrededor del 57\% de los participantes se concentran en edades de entre 31 y 58 años.

En la figura I se puede observar que las personas encuestadas dijeron cuidar su salud mediante la alimentación (88\%) y haciendo ejercicio (29\%). Esta tendencia se pudo apreciar también en las sesiones de grupo, en las que se enfatizó que la buena alimentación se refiere a tener una dieta balanceada que incluya especialmente vegetales y frutas, las carnes en menor proporción y sobre todo blancas; además de tratar de consumir productos más saludables que tengan un bajo contenido de azúcar, grasa y sal. Por ejemplo, hicieron comentarios como: "Yo trato de comer sano, y el ejercicio mínimo es caminar", "tratar de comer cosas que sean más ligth, menos dulces, menos grasas, menos saladas",

Cuadro I. Distribución porcentual según sexo y lugar de la población muestral encuestada, $n=$ 193 (Costa Rica, 201 I).

\begin{tabular}{|c|c|c|}
\hline Sexo & \multicolumn{2}{|c|}{ Zona geográfica } \\
\hline & San Carlos & Gran Área Metropolitana \\
\hline Femenino & 81,44 & 80,21 \\
\hline Masculino & 18,56 & 19,79 \\
\hline
\end{tabular}

"comemos bastantes verduras, frutas, hortalizas; incluyo dentro de la alimentación ensaladas, verduras, las carnes pero poquito; no meto muchas grasas ni condimentos, cocino lo más natural posible, camino y tomo bastante agua; evito el azúcar, nada que sea en exceso". Un 16\% de los encuestados señaló otras formas de cuidar la salud, entre las cuales están: lavarse las manos, tomar medicamentos, visitar al médico, dormir bien, manejar adecuadamente el estrés, no tomar licor y no fumar. Además, el $4 \%$ de las personas dijo que no cuida su salud de forma alguna (figura I).

Para el consumo de embutidos no existe una relación entre este y el lugar de residencia de las personas encuestadas $\left(X^{2}=0,00 \mathrm{I} ; p=0,9725\right)$, como se refleja en la figura 2. Tanto en la GAM como en San Carlos
Cuadro 2. Distribución porcentual según edad de la población muestral encuestada, $n=193$ (Costa Rica, 201 I).

\begin{tabular}{|c|c|}
\hline Grupo de edad & Porcentaje (\%) \\
\hline $17-30$ años & 22,92 \\
\hline $31-44$ años & 27,08 \\
\hline $45-58$ años & 30,21 \\
\hline $59-72$ años & 12,50 \\
\hline $73-85$ años & 7,29 \\
\hline
\end{tabular}

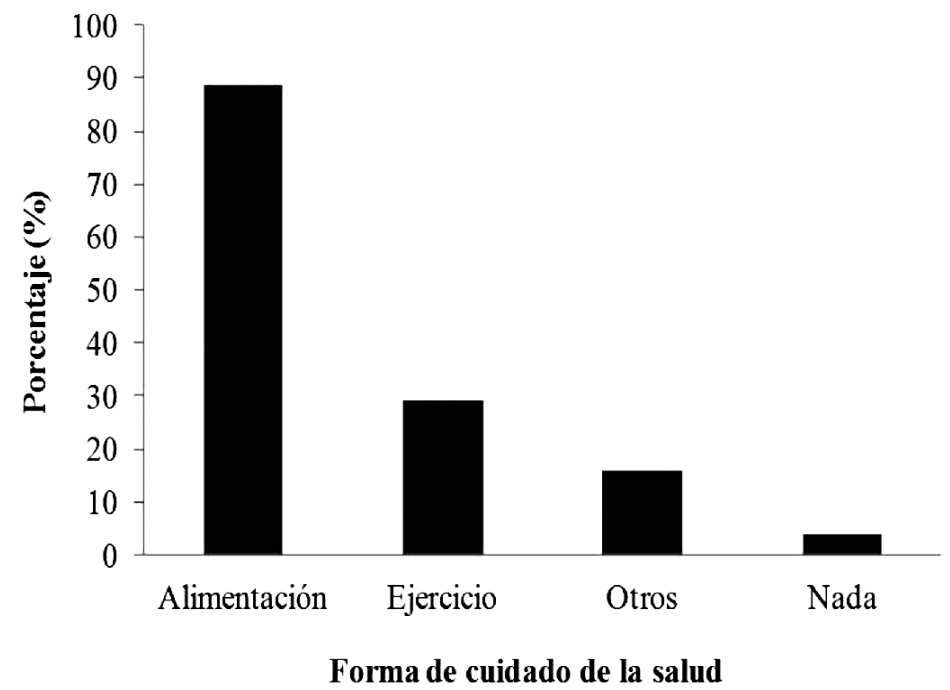

Figura I. Distribución porcentual de las alternativas de cuidado de la salud que practica la población muestral encuestada, $n=193($ Costa Rica, 201 I). 


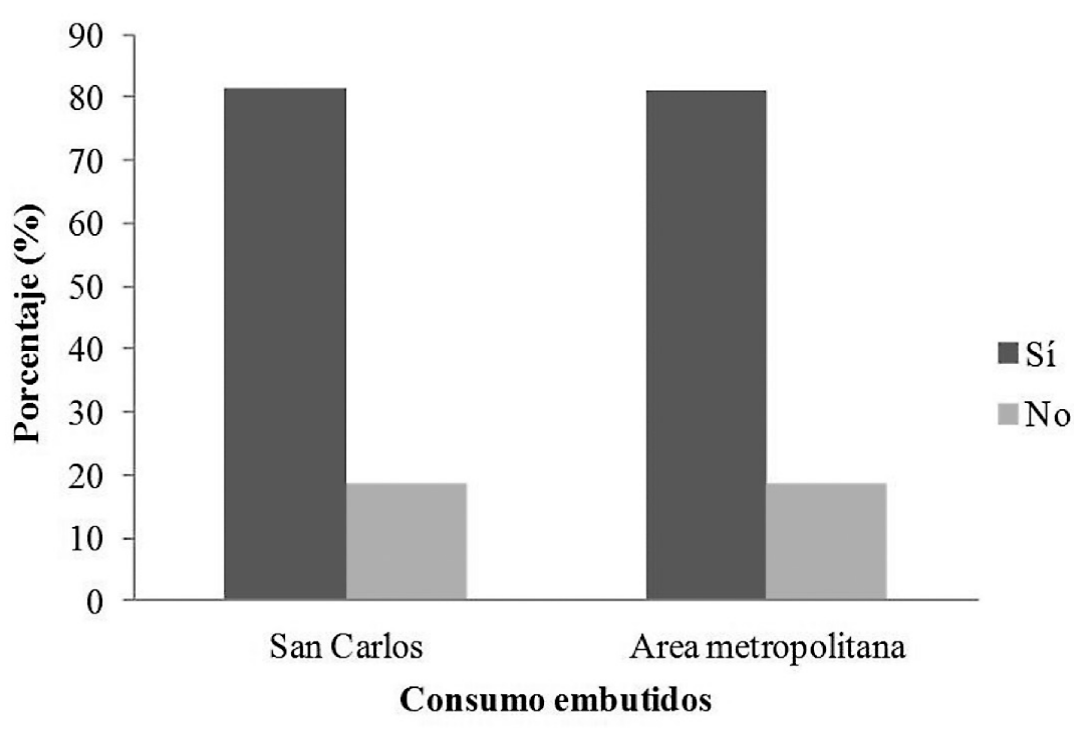

Figura 2. Distribución porcentual del consumo de embutidos de la población muestral encuestada, n =193 (Costa Rica, 2011).

existe alrededor de un 19\% de los encuestados que no ingieren estos productos. El $77 \%$ de las personas que no comen embutidos consideran que son malos para su salud porque contienen mucha grasa, o bien no los consumen por indicación médica. La segunda razón es porque no les gusta el producto, consideran que son elaborados a partir de recortes de carne y les desagrada el sabor.

La frecuencia de consumo de embutidos no mostró una relación con el lugar de residencia de la población estudiada $\left(X^{2}=4,192 ; p=0,6507\right)$. Así, alrededor del $30 \%$ de los encuestados consume embutidos dos veces por semana, el 20\% una vez por semana y el $18 \%$ menos de una vez por semana, de manera que aproximadamente solo el 30\% restante los ingiere más de tres veces por semana (figura 3a). En una encuesta telefónica sobre la alimentación en las diferentes clases sociales, realizada para conocer hábitos de consumo, se observó que el porcentaje de personas que consumía embutidos una vez por semana era 21,1\% (Calderón, Sandoval y Carrillo, 2000); muy similar a lo que se encontró para 20 I I. No obstante, en el año 2000 había un mayor número de personas que consumían estos productos menos de una vez por semana $(38,6 \%)$ (Calderón et al., 2000), lo cual a su vez evidencia que ha habido un aumento en la frecuencia de ingesta. En las sesiones de grupo, alrededor de la mitad de los participantes consumía embutidos entre dos y tres veces por semana, mientras que la otra parte los incluía en su alimentación más de tres veces por semana. De igual manera, no se encontró ninguna relación entre la frecuencia de consumo y el nivel académico $\left(X^{2}=34,565 ; p=0,2588\right)$ o el grupo de edad $\left(X^{2}=21,304 ; p=0,6208\right)$ de la población estudiada.

Por otro lado, al evaluar si la frecuencia de consumo tenía relación con el cuidado de la salud mediante la alimentación, se encontró una relación significativa $\left(X^{2}=28,093 ; p<0,000\right.$ I). Aproximadamente el 80\% de los encuestados que velan por su salud de esta forma consumen embutidos máximo dos veces por semana, mientras que un $55 \%$ de los que no cuidan su alimentación consumen estos productos más de tres veces por semana (figura 3b). Dado que los embutidos son percibidos como "malos para la salud", este comportamiento coincide con lo que se observó en un estudio realizado en Canadá, donde las personas que tenían mayor intención de consumir comida saludable eran los que usaban métodos de preparación de alimentos más saludables y adquirían comida no saludable con menor frecuencia (Mead, Gittelsohn, Roache y Sharma, 20 I0).

En relación con el tipo de embutido, los encuestados consumen mayoritariamente salchichón; el consumo 
de chorizo y otros embutidos (clasificación que incluye el paté y el salame), es relativamente bajo (figura 4a). Cabe mencionar que si bien en el estudio de Aguilar, Ivankovich, Figueroa y Fonseca (2003) el mayor consumo de embutidos se debió al salchichón, fue más consumida también la mortadela y el chorizo con respecto a las salchichas y el jamón; por lo que se nota un cambio en la preferencia de consumo. Es importante aclarar que en este estudio se consideró el jamón, a pesar de no ser un embutido, porque los consumidores no hacen la distinción.
No se encontró una relación entre el embutido de preferencia y el grupo de edad de la población estudiada $\left(X^{2}=40,844 ; p=0,0555\right)$, lo cual indica que la preferencia no parece estar relacionada con la edad. Por el contrario, sí se halló una relación entre el embutido de preferencia y el lugar de residencia de la población estudiada $\left(X^{2}=11,222 ; p=0,0472\right)$, la cual se puede apreciar en la figura 4b. En la GAM, las personas encuestadas prefieren consumir jamón, mientras que en San Carlos el embutido de preferencia es el salchichón. La preferencia por el consumo de jamón también fue observada en el
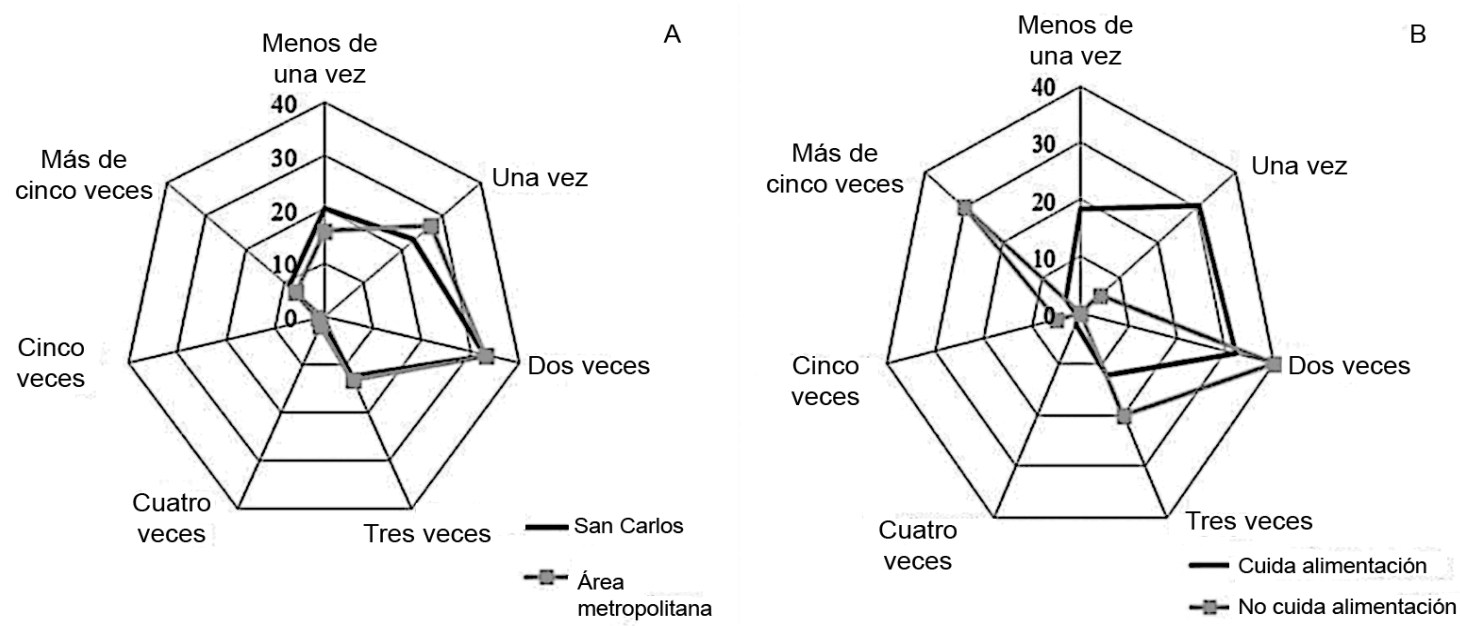

Figura 3. Frecuencia de consumo de embutidos según lugar de residencia (a) y cuidado de la salud por medio de la alimentación (b) en la población muestral encuestada, n= I57 (Costa Rica, 20 I I).
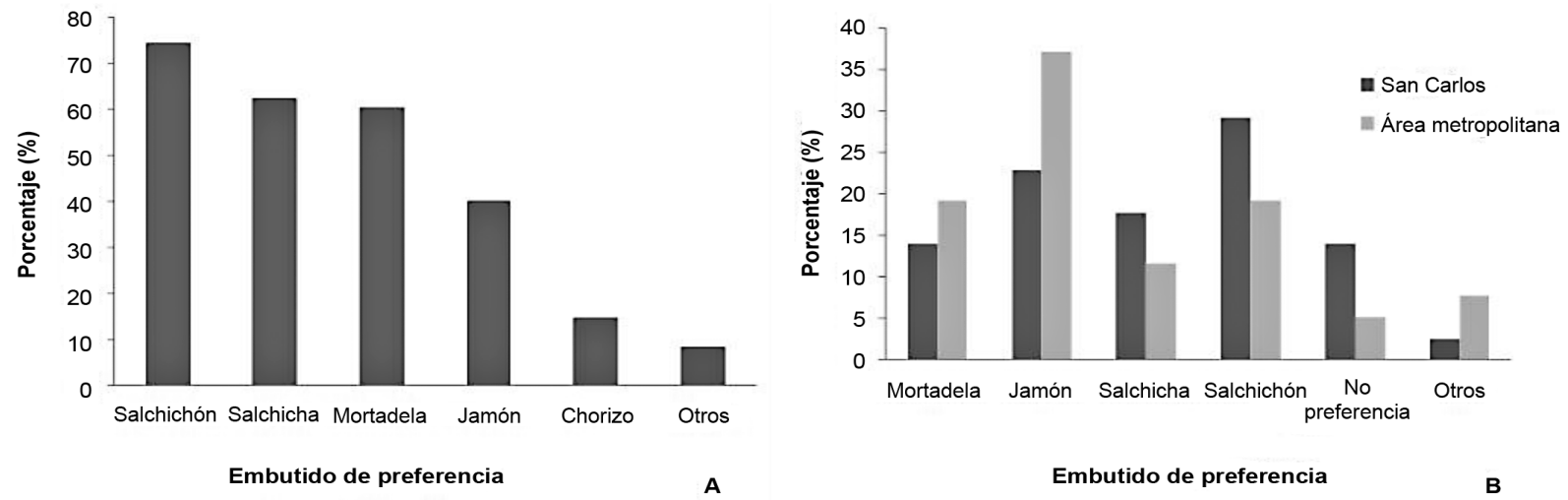

Figura 4. Distribución porcentual del tipo de embutido de preferencia para la población muestral (a), según lugar de residencia de la población muestral encuestada (b), n= 157 (Costa Rica, 201 I). 
estudio de Sáez, Aponte \& Castellano (2009). No obstante, cabe mencionar que en lo que respecta al salchichón, este fue uno de los embutidos menos preferidos por los consumidores venezolanos.

Las dos principales características de los embutidos por las cuales los encuestados adujeron consumirlos fueron su buen sabor (56\%) y el ser prácticos (26\%) (figura 5). Asimismo, en las sesiones de grupo estas características también fueron identificadas como las de mayor relevancia. Otra característica importante mencionada en las sesiones de grupo y que se complementa con la funcionalidad de los embutidos, es su versatilidad, pues, según indicaron los participantes, se pueden preparar de varias formas y acompañar con una amplia gama de alimentos. Incluso mencionaron que "los embutidos te pueden sacar de apuros", porque se puede preparar una comida en poco tiempo; y que además, "cuando se agotan las ideas para el almuerzo, con embutidos se resuelve". La disponibilidad de embutidos en diferentes puntos de venta y su variedad promueven el consumo. McCarthya y McCarthya (2007) señalan que, en el caso de los embutidos de cerdo, es bien conocida su popularidad, ya que se consideran sabrosos, versátiles y convenientes, características compatibles y deseables para los estilos de vida de los consumidores en la actualidad.
La versatilidad que fue señalada en las sesiones de grupo se puede relacionar con los modos de preparación de los embutidos, como se observa en la figura 6. Las personas encuestadas utilizan diferentes modos de preparación; el 68\% los preparan fritos, el $32 \%$ los usan crudos, es decir, sin ningún tipo de cocción en el hogar; el 22\% asados, el 21\% los prepara en salsa y el 7\% usa otras formas. Estas últimas incluyen cocinar en agua o en el microondas. Con respecto al lugar de residencia, las personas de San Carlos preparan los embutidos fritos $\left(X^{2}=\right.$ 12,116; $p=0,0005)$, mientras que la preparación "cruda" guarda mayor relación con la GAM $\left(X^{2}=\right.$ 10,846; $p=0,0010)$. En las sesiones realizadas en San Carlos, algunas participantes adujeron consumir los embutidos preparados de diferentes formas: "yo, de cualquier manera; me lo como hasta crudo"; "yo me los como así, crudos; solo el salchichón no". Se podría concluir que la preparación en forma de fritura relacionada con San Carlos se debe a la preferencia por el salchichón.

Asimismo, entre los alimentos con los que las personas encuestadas acostumbran acompañar los embutidos están las harinas en un 95\%, las legumbres en un $53 \%$, un $50 \%$ los complementa con vegetales y un $14 \%$ los ingiere con otros alimentos (figura 7). Cabe indicar que entre las harinas se mencionó: arroz, pan, tortillas, pasta y galletas de soda; entre las legumbres: frijoles y garbanzos; en relación con los

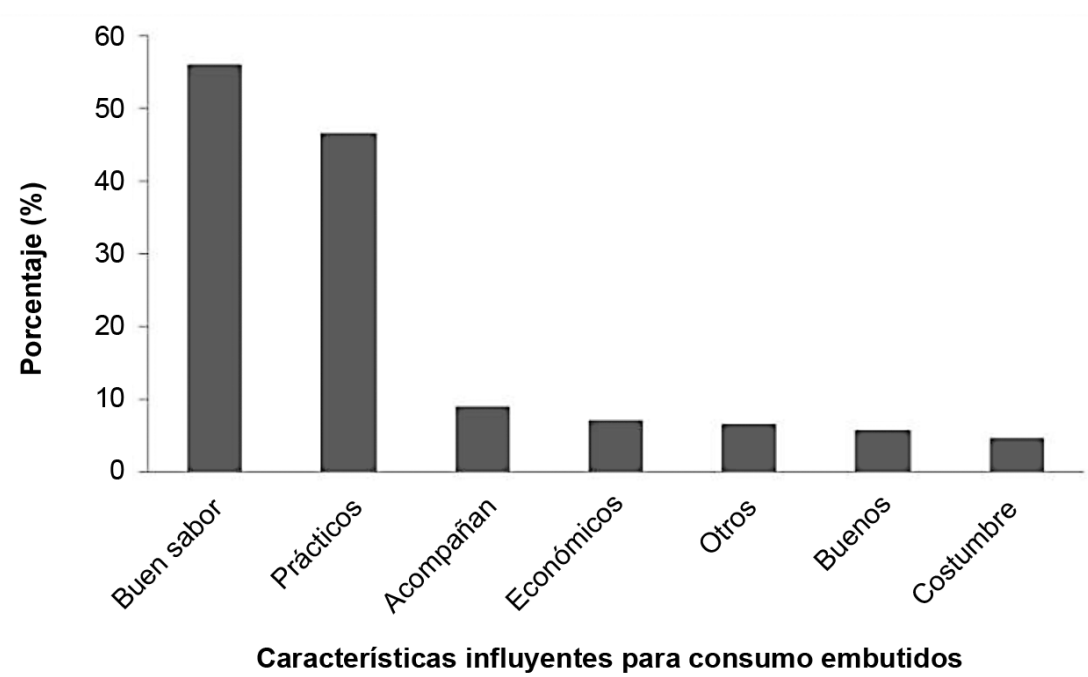

Figura 5. Distribución porcentual de las características de los embutidos por las que son consumidos por la población muestral encuestada, $n=157$ (Costa Rica, 201 I). 


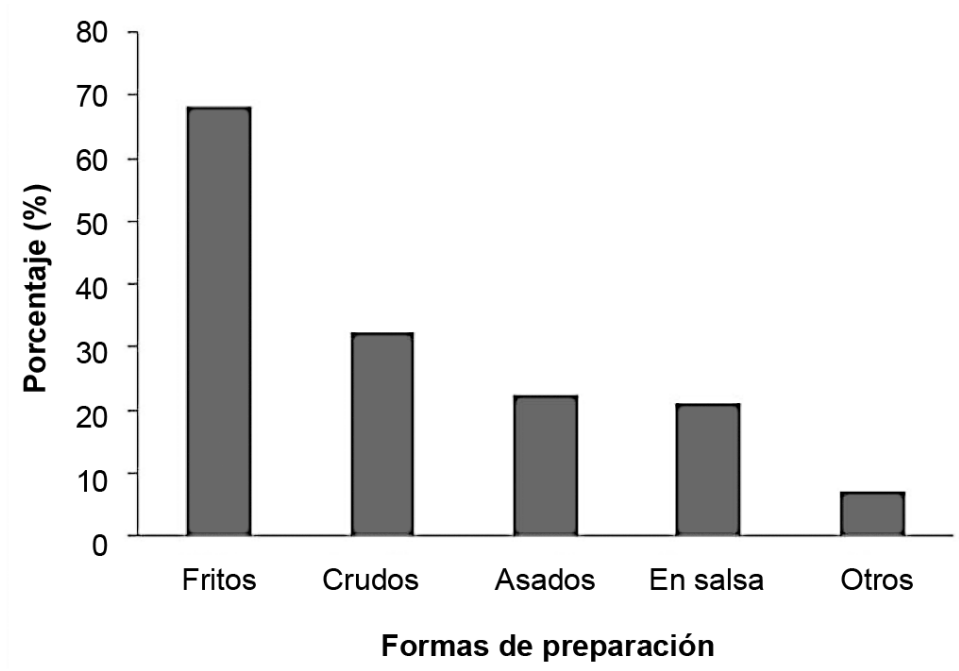

Figura 6. Distribución porcentual de las alternativas de preparación de los embutidos que practica la población muestral encuestada, $n=157$ (Costa Rica, 201 I).

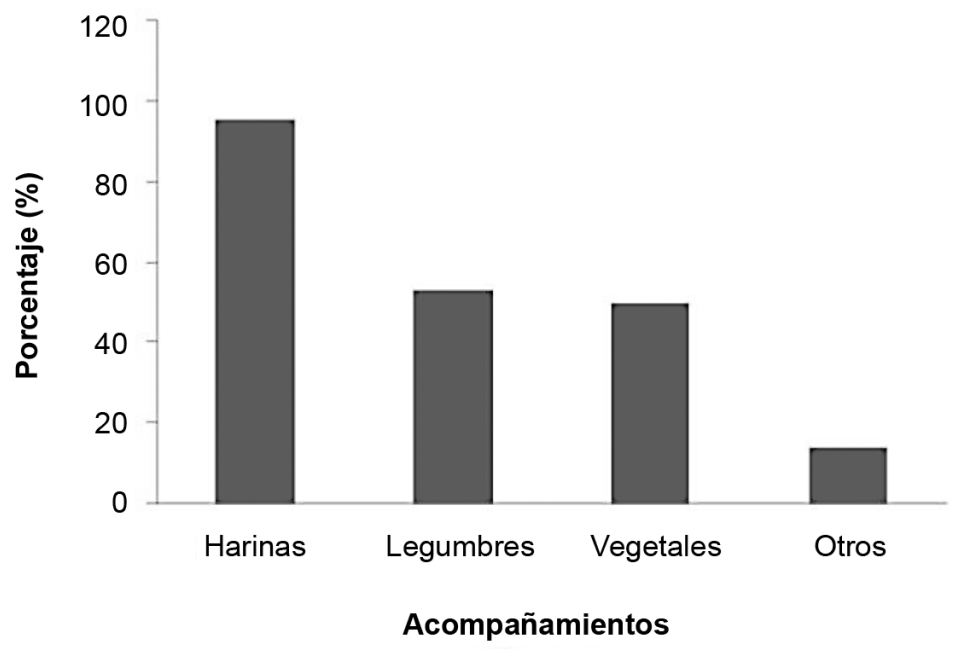

Figura 7. Distribución porcentual de las alternativas de acompañamientos de los embutidos que practica la población muestral encuestada, $n=157$ (Costa Rica, 201 I).

vegetales, estas personas acompañan los embutidos con papas y repollo; mientras que en la categoría de otros se incluyen quesos y huevo, principalmente.

En el cuadro 3 se pueden observar algunos de los platillos elaborados con los embutidos, según las formas de preparación y los acompañamientos utilizados por las personas que participaron en las sesiones de grupo. La variedad de formas de preparación y sus acompañamientos refleja que, en la realidad, los embutidos son un ingrediente que favorece la preparación de una diversidad de alimentos.
En la figura 8 se aprecia que los embutidos son consumidos en todos los tiempos de comida del día; un resultado similar al del estudio sobre las preferencias de los consumidores de embutidos venezolanos, en el que se evidenció que las personas ingieren estos productos al menos en una de sus comidas diarias (Sáez et al., 2009).

Asimismo, este comportamiento surgió también en las sesiones de grupo y se relacionó con lo prácticos que son, dado el poco tiempo y la baja dificultad que conlleva su preparación. En las sesiones se mencionó el consumo de embutidos en las fiestas 
Cuadro 3. Platillos elaborados con los embutidos según la forma de preparación y con los acompañamientos usados por las personas participantes en las sesiones de grupo.

\begin{tabular}{|c|c|c|}
\hline Forma de preparación & Platillos elaborados & Acompañamientos \\
\hline Crudos & $\begin{array}{c}\text { Emparedados } \\
\text { "Boquitas" con galleta de soda } \\
\text { Ensaladas }\end{array}$ & $\begin{array}{c}\text { Pan, galleta de soda, queso, } \\
\text { tomate, lechuga y salsas. }\end{array}$ \\
\hline Fritos o asados & $\begin{array}{c}\text { "Gallos" } \\
\text { Arroces } \\
\text { "Casados" } \\
\text { Tortas con huevo }\end{array}$ & $\begin{array}{c}\text { Tortilla, arroz, frijoles, huevo, } \\
\text { repollo, tomate y salsas. }\end{array}$ \\
\hline En salsa & $\begin{array}{c}\text { Pastas } \\
\text { Picadillos } \\
\text { Frijoles blancos } \\
\text { Lentejas } \\
\text { Garbanzos }\end{array}$ & Fideos, verduras, lentejas, \\
garbanzos, frijoles blancos y salsas.
\end{tabular}

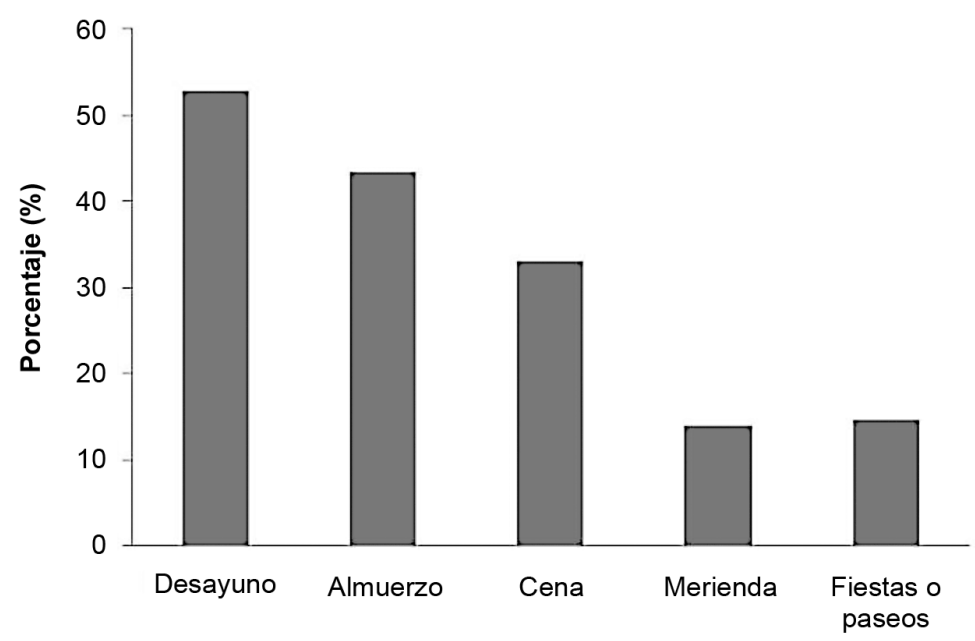

Situaciones de consumo

Figura 8. Distribución porcentual de las situaciones relacionadas con el consumo de los embutidos por la población muestral encuestada, $n=157$ (Costa Rica, 201 I).

o actividades familiares: "en las fiestas siempre hay salchichón", "los famosos gallitos"; se destacó que es algo que no puede faltar en la parrilla, como merienda o "para picar". Los participantes apoyan que su uso se debe a la funcionalidad y a que no se requieren conocimientos de cocina para prepararlos; "generalmente, cuando uno está picando, lo que busca es un embutido, fácil de preparar, rápido; cualquiera puede preparar algo con un embutido".
Los supermercados (74\%) son el principal lugar de compra donde los encuestados adquieren los embutidos, seguidos por las carnicerías (18\%) y las pulperías (9\%). Según Aguilar y colaboradores (2003), el lugar de compra para la mortadela, las salchichas y el jamón es el supermercado, mientras que el en el caso del salchichón, el chorizo y el paté son los lugares sin autoservicio. De acuerdo con las sesiones grupales, este mayor porcentaje de compra 
en los supermercados puede atribuirse a la disponibilidad de horarios y a la posibilidad de comprar otros productos complementarios en el mismo lugar (figura 9). Esto coincide con el estudio realizado por Sáez y colaboradores (2009) en Venezuela, donde se apreció que los supermercados estaban entre las primeras alternativas de lugares donde los consumidores compraban los embutidos, y debido en mayor medida a la variedad de productos que ofrecen.

Con respecto a la marca de mayor consumo, es de relevancia indicar que no existió una interacción entre esta y el lugar de residencia de las personas encuestadas $\left(X^{2}=10,087 ; p=0,259\right)$; sin embargo, la tendencia observada es que el $25 \%$ de los encuestados de la GAM consume la marca Cinta Azul, el I 8\% no sabe la marca de los embutidos que ingiere, el I3\% compra Zar, el I I\% Kimby y el 9\% otras marcas como Premier y Kali; mientras que en San Carlos el $27 \%$ no sabe cuál es la marca de embutidos que más consume, el I $8 \%$ consume mayor cantidad de embutidos Cinta Azul, el I5\% otros marcas (entre las que se incluye Hidalgo, que es fabricada en el cantón), el I I\% no tiene preferencia por una marca en particular y el 8\% consume Zaragoza (figura 10). Según Aguilar y colaboradores (2003), la última marca que habían comprado las encuestadas al momento del estudio, para prácticamente todos los tipos de embutidos evaluados, fue Cinta Azul, a excepción del chorizo, el cual carecía de marca, por lo que se puede ver que la marca Cinta Azul continúa teniendo cierta posición en el mercado. Es importante destacar que $18 \%$ de personas de la GAM y $27 \%$ de San Carlos dijeron no saber la marca de mayor consumo, junto con el II\% de San Carlos que dijo no tener una marca preferida. En las sesiones de grupo algunas personas comentaron: "yo no me fijo en la marca", "uno va cambiando", "depende de las ofertas", "uno prueba y decide si le gusta"; por lo que aún existe espacio en el mercado para posicionar una marca cuyo competidor más popular sería Cinta Azul.

\section{Conclusiones}

En la población encuestada no se encontraron diferencias significativas en los hábitos entre los consumidores de embutidos de la GAM y San Carlos, excepto para el embutido de preferencia, dado que mientras en la GAM es el jamón, en San Carlos es el salchichón.

Aproximadamente el $80 \%$ de la población total encuestada consume embutidos, independientemente de que residan en la GAM o en San Carlos.

Los encuestados consumen los embutidos dentro de cualquiera de sus comidas del día. Además, el lugar de compra mayoritariamente es el supermercado, seguido por carnicerías y pulperías.

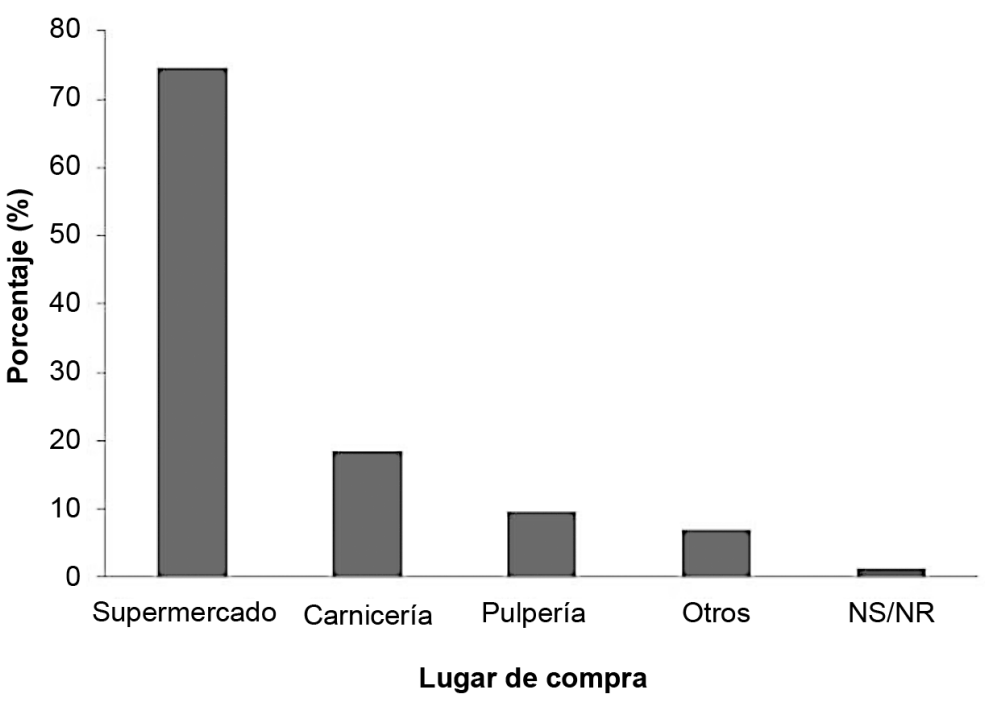

Figura 9. Distribución porcentual de las alternativas de lugares en que compra los embutidos la población muestral encuestada, $n=157$ (Costa Rica, 20 I I). 


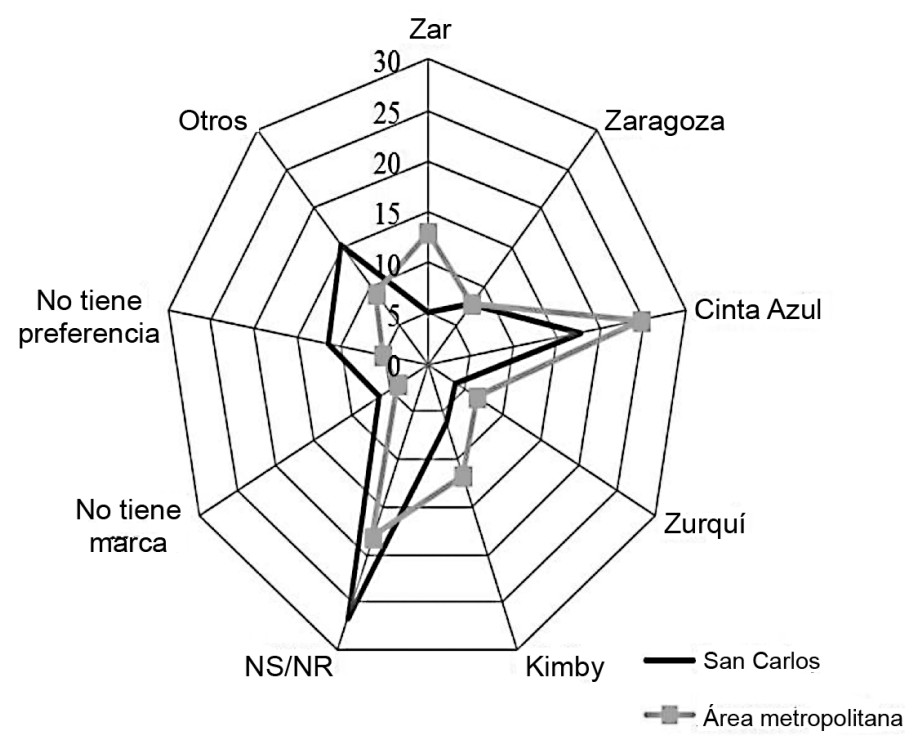

Figura 10. Distribución porcentual de la marca de embutido de mayor consumo según lugar de residencia de la población muestral encuestada, $n=157$ (Costa Rica, 201 I).

Las principales motivaciones por las que se consumen embutidos son su buen sabor y lo prácticos que son; a esto se agrega su versatilidad a la hora de preparar las comidas.

La población encuestada acostumbra preparar los embutidos principalmente fritos, "crudos" (sin aplicar un método de cocción en el hogar), asados y en salsa. La versatilidad de los embutidos también permite acompañar alimentos como harinas, legumbres y verduras.

Entre los embutidos que más consumen las personas encuestadas están el salchichón, las salchichas, la mortadela, el jamón y el chorizo. En general, la marca mencionada como de mayor preferencia por la población estudiada es Cinta Azul.

El porcentaje de la población estudiada que no consume embutidos se explica por dos razones: primero, porque percibe que son elaborados a partir de recortes cárnicos y además les desagrada el sabor; y segundo, considera que son malos para la salud por el alto contenido de grasa percibido o porque algún profesional de la salud se los prohibió.

La frecuencia de consumo de la mayor parte de las personas encuestadas es de dos veces por semana. Esta misma frecuencia de consumo está presente en el $80 \%$ de las personas que cuidan su salud mediante la alimentación.
Para los industriales del sector cárnico, el aporte novedoso de esta investigación reside en poder identificar la importancia que tiene para el consumidor conocer con más detalle los aspectos de calidad relacionados con los embutidos y enfocarse en la materia prima utilizada, con el fin de generar confianza en el consumidor que cuida su salud, que podría aumentar su consumo al menos una vez más por semana. Se recomienda un estudio cualitativo para valorar los mensajes sobre la calidad del producto que se impulsará y hacer los ajustes necesarios y específicos según la percepción de los consumidores.

\section{Agradecimientos}

Esta investigación se realizó gracias al apoyo financiero del Sistema de Estudios de Posgrado de la Universidad de Costa Rica. Se agradece a todas las personas que participaron en las entrevistas, las sesiones focales y la encuesta.

\section{Bibliografía}

Aguilar, F., Ivankovich, C., Figueroa, J. \& Fonseca, A. (2003). Estudio sobre el consumo de productos cárnicos en Costa Rica. San José: Centro Nacional de Ciencia y Tecnología de Alimentos. 
Barrios, E. \& Costell, E. (2004). Review: Use of methods of research into consumers opinions and attitudes in food research. Food Science and Technology International, 10(6), 359-37।.

Bellenger, D., Bernhardt, K. \& Goldstucker, J. (1976). Qualitative Research Techniques: Focus Groups Interviews. Qualitative Research in Marketing. Chicago: American Marketing Association (pp. 7-28). En T. Hayes y C. Tathum. 1989. Focus Group Interviews: A Reader (pp. 10-25). US: American Marketing Association.

Benassini, M. (200 I). Introducción a la investigación de mercados: un enfoque para América Latina. México: Pearson Educación.

Calderón, O., Sandoval, I. \& Carrillo, M. (2000). La población costarricense frente a la crisis, sus vivencias políticas y la actividad turística del país. IDESPO. Obtenido de http://wrw. nacion.com/In_ee/ESPECIALES/una/idespo3/I .html

Guillén, I. (2007). Propuesta de investigación preliminar sobre el Mercado de los Embutidos. MEIC. Obtenido de http://reventazon.meic.go.cr/informacion/cpc/2007//O-002-07.pdf

Fern, E. (febrero, 1982). Use of focus group for idea generation: The effects of group size, acquaintanceships, and moderator on response quantity and quality. Journal of Marketing Research, 19, 1-13.

Freitas, H., Oliveira, M., Jenkins, M. \& Popjoy, O. (1998). The Focus Group, a qualitative research method (Working Paper No. 0 I0298). Escola de Administraçao da Universidade Federal do Rio Grande do Sul. Obtenido de http://www.ea.ufrgs.br/ professores/hfreitas/files/artigos/1998/1998_079_ISRC.pdf

Gordon, R. \& Goldsmith, E. (1994). Consumer Psychology for Marketing. London: Routledge.
Hill, C. \& Jones, G. (2005). Administración Estratégica: un enfoque integrado. 6 ed. México: McGraw Hill.

Hoyer,W. \& Maclnnis, D. (20 I0). Comportamiento del Consumidor. 5 ed. México: Cengage Learning.

Ivankovich-Guillén, C.\& Araya-Quesada,Y. (20 I I)."Focus groups: Técnica de investigación cualitativa en investigación de mercados. Ciencias Económicas, 29( I), 545-554.

Kotler, P. \& Keller, K. (2006). Dirección de marketing. 12 ed. México: Pearson Educación.

McCarthya, M. \& McCarthya, P. (2007). Parents' Perceptions of Pork Sausages as a Meal Solution. Journal of Food Products Marketing, I 3(49), I-22.

Mead, E., Gittelsohn, J., Roache, C. \& Sharma, S. (20I0). Healthy food intentions and higher socioeconomic status are associated with healthier food choices in an Inuit population. Journal of Human Nutrition and Dietetics, 23(I), 83-91.

Meilgaard, M., Vance, G. \& Carr, T. (2007). Sensory Evaluations Techniques. 4 ed. Florida:Taylor \& Francis.

Pope, J. (2002). Investigación de mercados: guía maestra para el profesional. Bogotá: Norma.

Sáez, A., Aponte, B. \& Castellano, S. (2009). Preferencias del consumidor de embutidos en el municipio Maracaibo del Estado Zulia, Venezuela. Agroalimentaria, 15(29), 55-67.

Seymour, D. (1988). Marketing Research: Qualitative Methods for the Marketing Professional. US: Probus Publishing Company.

Sutherland, K. (1991). New product development. 2 ed. En Guilfords y Kings, L. Researching business markets: The IMRA handbook of business-to-business marketing research (pp. 341 350). Inglaterra: Baddles Ltd. 